\title{
Benefícios do condicionamento cardiorrespiratório em crianças ou adolescentes com paralisia cerebral
}

\author{
Benefits of cardiorespiratory training in children or adolescents \\ with cerebral palsy
}

\section{Kérima Giamarim Batista ${ }^{[a]}$, Priscila de Oliveira Lopes ${ }^{[b]}$, Simone Mitie Serradilha ${ }^{[c]}$, Grazielle Aurelina Fraga de Souza ${ }^{[\mathrm{d}]}$, Geruza Perlato Bella ${ }^{[\mathrm{e}]}$, Regina Célia Turrola de Souza ${ }^{[\mathrm{f}]}$}

[a] Fisioterapeuta graduada pela Pontifícia Universidade Católica de Minas Gerais (PUCMinas), especialista em Fisioterapia Aplicada à Neurologia Infantil pela Universidade Estadual de Campinas (Unicamp), Campinas, SP - Brasil, e-mail:kgbatistafisio@hotmail.com

[b] Fisioterapeuta graduada pela Pontifícia Universidade Católica de Minas Gerais (PUCMinas), especialista em Fisioterapia Aplicada à Neurologia Infantil pela Universidade Estadual de Campinas (Unicamp), Campinas, SP - Brasil, e-mail: priolop19@hotmail.com

[c] Fisioterapeuta graduada pela Pontifícia Universidade Católica de Minas Gerais (PUCMinas), especialista em Fisioterapia Aplicada à Neurologia Infantil pela Unicamp, supervisora do Curso de Especialização em Fisioterapia Aplicada à Neurologia Infantil da Universidade Estadual de Campinas (Unicamp), Campinas, SP - Brasil, e-mail: siserradilha@yahoo.com.br

[d] Fisioterapeuta graduada pela Pontifícia Universidade Católica de Minas Gerais (PUCMinas), especialista em Fisioterapia Aplicada à Neurologia Infantil pela Universidade Estadual de Campinas (Unicamp), Campinas, SP - Brasil, e-mail:grazielle_fraga@hotmail.com

[e] Fisioterapeuta, Mestre em Biologia Molecular e Funcional - Fisiologia, pelo Instituto de Biologia da Unicamp, supervisora do Curso de Especialização em Fisioterapia Aplicada à Neurologia Infantil da Universidade Estadual de Campinas (Unicamp), Campinas, SP - Brasil, e-mail: geruza@fcm.unicamp.br

[f] Fisioterapeuta, Doutora em Neurociências pelo departamento de Neurologia da Faculdade de Ciências Médicas da Unicamp, coordenadora do Curso de Especialização em Fisioterapia Aplicada à Neurologia Infantil da Universidade Estadual de Campinas (Unicamp), Campinas, SP - Brasil, e-mail: turrola@fcm.unicamp.br

\section{Resumo}

Introdução: $O$ treinamento cardiorrespiratório tem mostrado efeitos positivos para a população com paralisia cerebral, melhorando a capacidade funcional e reduzindo o gasto energético e os riscos cardiovasculares. Objetivo: Investigar os efeitos do treinamento aeróbico na pressão arterial, distância percorrida e no consumo máximo de oxigênio. Métodos: Participaram do estudo oito crianças e adolescentes com paralisia cerebral do tipo hemiparética e diparética espástica (G1) e oito do grupo controle (G2), pareados por sexo e idade. Todos foram avaliados pelo teste de Cooper, no qual foi aferida a pressão arterial inicial e final, distância percorrida e cálculo do $\mathrm{VO}_{2}$ máx. O G1 participou de um programa que constava de alongamentos e treinamento em esteira durante 24 sessões. Resultados: Houve melhora 
significativa após o treinamento do $\mathrm{G} 1$ na distância percorrida, $\mathrm{VO}_{2}$ máx e pressão arterial sistólica inicial. Na comparação entre grupos observou-se diferença estatisticamente significativa na distância percorrida e $\mathrm{VO}_{2}$ máx pré e pós-tratamento, pressão arterial sistólica (PAS) inicial pós e PAS final pós-tratamento. Após o período de destreinamento, não houve perda significativa da melhora nas variáveis do G1, exceto do $\mathrm{VO}_{2}$ máx. Conclusão: $\mathrm{O}$ treinamento aeróbico pode trazer benefícios cardiorrespiratórios para a população com paralisia cerebral do tipo hemiparética e diparética espástica, podendo ser incluído dentro de um programa de reabilitação.

Palavras-chave: Paralisia cerebral. Hemiparético. Diparético. Condicionamento cardiorrespiratório.

\begin{abstract}
Introduction: The cardiorespiratory training has revealed positive effects on the population with cerebral palsy, improving functional capacity, reducing the energy cost and cardiovascular risks. Objective: The aim of this study was to investigate the effects of aerobic training on blood pressure, ran/walked distance and maximal oxygen consumption. Methods: The sample was composed by eight children and adolescents with hemiparetic and diparetic spastic cerebral palsy (G1) and eight children in control group (G2), matched on gender and age. They were assessed through the Cooper test, measuring initial and final blood pressure, ran/ walked distance and $\mathrm{VO}_{2}$ max. G1 participated in a program with stretching and treadmill training during 24 sessions. Results: There was significant improvement for $G 1$ after training on ran/walked distance, $V_{2}$ max and initial systolic arterial pressure (SAP). When comparing the two groups, a statistically significant difference was observed on the ran/walked distance and $\mathrm{VO}_{2}$ max before and after training, systolic arterial pressure (SAP) before and after training. After the detraining period, there was no significant decay on the improvement of $\mathrm{G} 1$ variables, except for $\mathrm{VO}_{2}$ max. Conclusion: The aerobic training may bring cardiorespiratory benefits to the population with hemiparetic and diparetic spastic cerebral palsy; therefore, it can be included in a rehabilitation program.
\end{abstract}

Keywords: Cerebral palsy. Hemiparetic. Diparetic. Cardiorespiratory training.

\title{
Introdução
}

A Paralisia Cerebral (PC), ou Encefalopatia Crônica, é caracterizada como um grupo não progressivo, mas frequentemente mutável, de distúrbios motores, secundário à lesão do Sistema Nervoso Central (SNC) em desenvolvimento, ou seja, desde a fase embrionária até os 2 anos de vida extrauterina (1-3).

O quadro clínico é determinado principalmente por alterações de tônus muscular e postura (4), fraqueza muscular e baixa resistência à atividade física (5). A associação desses fatores pode gerar consequências funcionais e sociais, tendendo o paciente a ficar cada vez mais sedentário e isolado socialmente.

A baixa resistência aeróbica observada nesses indivíduos pode ser atribuída à diminuição do recrutamento de unidades motoras durante atividade dinâmica, redução da capacidade oxidativa dos músculos paréticos e diminuição global da resistência aeróbica, com aumento do gasto energético durante a realização de atividades da vida diária e exercícios submáximos $(6,7)$.

Estudos comprovam que exercícios aeróbios têm mostrado efeitos positivos em pacientes hemiplégicos crônicos, como a redução da atrofia por desuso, por meio de maior recrutamento de unidades motoras; ganhos na capacidade funcional, com menor gasto energético nas atividades de vida diária (AVDs), e menor risco cardiovascular, com benefícios no controle da pressão arterial (PA) e da frequência cardíaca $(\mathrm{FC})(8,9)$.

O treinamento aeróbio para hemiparéticos crônicos tem alto potencial na diminuição da morbidade e mortalidade, além de aumentar a independência física e a qualidade de vida. E apesar da evidência dessa população ser fisicamente descondicionada, os profissionais da área de saúde têm receio de utilizar esse 
tipo de tratamento dentro da reabilitação, possivelmente por causa do risco de quedas, lesões pelo exercício repetido e desconhecimento de que o treinamento pode reduzir o alto gasto energético e a demanda cardiovascular $(6,10)$.

Visto que crianças e adolescentes com paralisia cerebral apresentam-se fisicamente descondicionados, em decorrência das alterações motoras já citadas acima, e que programas de condicionamento cardiorrespiratório têm se mostrado benéficos para essa população (11), este estudo tem como objetivo propor um protocolo de treinamento em esteira para avaliar os efeitos do condicionamento cardiorrespiratório nesses indivíduos.

\section{Método}

Neste estudo participaram 16 crianças ou adolescentes de ambos os sexos, com idade média de 9,6 4 2,4 anos, divididos em dois grupos. No grupo experimental 1 (G1) participaram oito indivíduos com paralisia cerebral do tipo hemiparética e diparética espástica, com marcha independente, selecionados no Ambulatório de Fisioterapia em Neurologia Infantil do Hospital de Clínicas da Universidade Estadual de Campinas. No grupo controle 2 (G2) participaram oito indivíduos sem alterações neurológicas, pareados com os sujeitos do grupo 1 em relação à idade e sexo, e sem prática de atividade física regular (exceto atividades escolares na disciplina de Educação Física), selecionados aleatoriamente. Todos foram informados a respeito dos procedimentos e assinaram um termo de consentimento livre e esclarecido. A pesquisa foi aprovada pelo Comitê de Ética e Pesquisa da Faculdade de Ciências Médicas da Unicamp, de acordo com a resolução n. 196 de 10 de outubro de 1996, tendo sido registrada sob o número 592/2006. Este estudo caracteriza-se como ensaio clínico controlado.

Os indivíduos do G1 foram submetidos a um treinamento cardiorrespiratório em esteira ergométrica e as avaliações (distância percorrida, pressão arterial e consumo máximo de oxigênio) foram feitas em três momentos durante a pesquisa: antes do inicio do treinamento (avaliação pré-treinamento); ao fim do treinamento (avaliação pós-treinamento); e 12 semanas após o término do treinamento (reavaliação após período de destreinamento).

A distância percorrida (DiP) foi avaliada utilizando o teste de 12 minutos, ou de Cooper, que consiste em correr ou andar durante 12 minutos consecutivos, tentando percorrer a maior distância possível. Deve ser realizado em local com superfície dura e lisa, com vestimenta adequada e no mínimo 2 horas após a refeição. É utilizado para crianças a partir de 7 anos, pois mede a condição aeróbica com maior fidedignidade que testes mais curtos (12). Foi realizado em um percurso de 18 metros, demarcado a cada 3 metros, com terapeuta ao lado da criança e monitoramento do PA no início e no fim do teste. Todos os indivíduos foram encorajados verbalmente com frases padronizadas de incentivo a cada minuto do teste.

O consumo máximo de oxigênio foi calculado antes e ao fim do treinamento cardiorrespiratório, através da equação de Cooper:

$$
\mathrm{VO}_{2} \text { máx: }\left(\mathrm{ml} \mathrm{x} \mathrm{Kg}^{-1}\right)=\mathrm{D}(\mathrm{m})-504,1 / 44,8
$$

Após avaliação pré-treinamento, o G1 iniciou o protocolo de treinamento em esteira ergométrica Moviment Treadmill Cardio fit innovation ${ }^{\mathrm{TM}}$, num total de 24 sessões com duração de aproximadamente 40 minutos, duas vezes por semana e em dias alternados, sendo divididos em duas etapas: 20 minutos de treinamento em esteira e 10 minutos iniciais e finais de alongamento passivo dos músculos flexores de cotovelo, flexores de punho, flexores de dedos, flexores, adutores e extensores de quadril, extensores de joelho e plantiflexores mantidos por 30 segundos (13) .

A primeira sessão de treinamento em esteira foi desconsiderada do protocolo de tratamento com objetivo de familiarização com a terapia proposta, sendo colocada a carga mínima de $2,5 \mathrm{~km} / \mathrm{h}$.

Por medida de segurança, a FC foi monitorada durante todo o treinamento em esteira, de modo que fosse interrompido caso ela atingisse $80 \%$ da FC máxima. 
Após o término das 24 sessões de treinamento, os pacientes do G1 voltaram à rotina de atividades normais, sendo orientados a não realizar atividades físicas além do que era habitual antes do início do treinamento cardiorrespiratório. Após 12 semanas de período de destreinamento, foram reavaliados (distância percorrida, pressão arterial e consumo máximo de oxigênio).

O G2 foi submetido ao mesmo protocolo de avaliação em uma medida transversal, para obtenção de parâmetros de sujeitos sem alterações neurológicas e comparações entre grupos.

$\mathrm{Na}$ análise de resultados, os dados foram armazenados no programa SPSS ${ }^{\circledR}$, versão 7.5, com a utilização do teste de Wilcoxon para dados pareados e o teste de Mann Whitney, para variáveis entre grupos.

\section{Resultados}

Na Tabela 1 encontram-se as variáveis descritivas do grupo com paralisia cerebral, do qual participaram oito sujeitos com média de idade de 9,6 \pm 2,5 anos, sendo cinco hemiparéticos e três diparéticos. Por intercorrências clínicas, um sujeito não completou o protocolo de treinamento, realizando apenas um total de 14 sessões. Constata-se que todos os sujeitos obtiveram melhora no tempo máximo de treino e na velocidade máxima da esteira, inclusive aquele que não completou o total de sessões propostas.

Tabela 1 - Variáveis descritivas e dados comparativos do treinamento do grupo com paralisia cerebral

\begin{tabular}{ccccccccc}
\hline Sujeitos & Sexo & $\begin{array}{c}\text { Idade } \\
(\text { anos })\end{array}$ & $\begin{array}{c}\text { Diagnóstico } \\
\text { Disfuncional }\end{array}$ & Sessões & $\begin{array}{c}\text { T.M.T.I } \\
(\mathbf{m i n})\end{array}$ & $\begin{array}{c}\text { T.M.T.F } \\
(\mathbf{m i n})\end{array}$ & $\begin{array}{c}\text { V.M.I.E } \\
(\mathbf{k m} / \mathbf{h})\end{array}$ & $\begin{array}{c}\text { V.M.F.E } \\
(\mathbf{k m} / \mathbf{h})\end{array}$ \\
\hline 1 & $\mathrm{M}$ & 11 & $\mathrm{D}$ & 24 & 10 & 20 & 2,5 & 4,5 \\
2 & $\mathrm{M}$ & 14 & $\mathrm{H}$ & 24 & 20 & 20 & 2,5 & 4,2 \\
3 & $\mathrm{M}$ & 7 & $\mathrm{D}$ & 24 & 20 & 20 & 2,5 & 4,0 \\
4 & $\mathrm{M}$ & 11 & $\mathrm{H}$ & 24 & 20 & 20 & 2,5 & 5,0 \\
5 & $\mathrm{M}$ & 7 & $\mathrm{D}$ & 24 & 5 & 20 & 2,5 & 2,9 \\
6 & $\mathrm{M}$ & 8 & $\mathrm{H}$ & 24 & 10 & 20 & 2,5 & 5,2 \\
7 & $\mathrm{~F}$ & 10 & $\mathrm{H}$ & 24 & 10 & 20 & 2,5 & 3,7 \\
8 & $\mathrm{~F}$ & 9 & $\mathrm{H}$ & 14 & 10 & 20 & 2,5 & 3,4 \\
\hline
\end{tabular}

Nota: $\mathrm{M}=$ masculino; $\mathrm{F}=$ feminino; $\mathrm{D}=$ diparético; $\mathrm{H}=$ hemiparético; T.M.T.I= tempo máximo de treino inicial; T.M.T.F= tempo máximo de treino final; V.M.I.E= velocidade máxima inicial na esteira; V.M.F.E= velocidade máxima final na esteira.

Os valores pré e pós-tratamento de distância percorrida (DiP), consumo máximo de oxigênio $\left(\mathrm{VO}_{2}\right.$ máx), pressão arterial sistólica (PASi) e diastólica (PADi) de repouso, pressão arterial sistólica (PASf) e diastólica (PADf) final do grupo com paralisia cerebral encontram-se na Tabela 2. Nela se verifica melhora significativa na DiP, $\mathrm{VO}_{2}$ máx, PASi e PASf e melhora não significativa, mas clinicamente relevante, nas demais variáveis. No pós-treinamento, a melhora da $\mathrm{DiP}$ e $\mathrm{VO}_{2}$ máx resulta em aproximação estatística dos valores obtidos pelo grupo controle, e a redução da PASi e da PASf no grupo com PC aumenta significativamente a diferença entre os grupos (Tabela 3). 
Tabela 2 - Mínima, média, desvio padrão e máxima dos valores de DiP, $\mathrm{VO}_{2}$, PASi, PADi, PASf e PADf pré e pós treinamento do grupo com paralisia cerebral

\begin{tabular}{|c|c|c|c|c|c|c|c|}
\hline & \multicolumn{3}{|c|}{ Pré-tratamento } & \multicolumn{3}{|c|}{ Pós-tratamento } & \multirow[t]{2}{*}{$\mathrm{p}$} \\
\hline & Mínima & Média/DP & Máxima & Mínima & Média/DP & Máxima & \\
\hline DiP & 288 & $681 \pm 256,5$ & 927 & 465 & $930,7 \pm 264,5$ & 1242 & $0,012^{*}$ \\
\hline $\mathrm{VO}_{2}$ & $-4,4$ & $3,9 \pm 5,7$ & 9,4 & 0,9 & $9,7 \pm 5,5$ & 16,5 & $0,012^{*}$ \\
\hline PASi & 90 & $100 \pm 10,7$ & 120 & 80 & $88,7 \pm 8,3$ & 100 & $0,024^{*}$ \\
\hline PADi & 60 & $66,2 \pm 10,6$ & 90 & 50 & $60 \pm 9,2$ & 70 & $0,129^{\mathrm{NS}}$ \\
\hline PASf & 90 & $110 \pm 11,9$ & 130 & 80 & $102,5 \pm 15,8$ & 120 & $0,262^{\mathrm{NS}}$ \\
\hline PADf & 60 & $68,7 \pm 8,3$ & 80 & 50 & $63,7 \pm 11,9$ & 80 & $0,33^{\mathrm{NS}}$ \\
\hline
\end{tabular}

Wilcoxon não paramétrico *: diferenças estatisticamente significativas $\mathrm{p}<0,05$, NS: não significativo. $\mathrm{DiP}=$ distância percorrida; $\mathrm{VO}_{2}=$ consumo máximo de oxigênio; $\mathrm{PASi}=$ pressão arterial sistólica inicial; $\mathrm{PADi}=$ pressão arterial diastólica inicial; $\mathrm{PASf}=$ pressão arterial sistólica final; PADf= pressão arterial diastólica final.

Tabela 3 - Mínima, média, desvio padrão e máxima dos valores de DiP, $\mathrm{VO}_{2}$, PASi, PADi, PASf e PADf pré e pós treinamento do grupo com paralisia cerebral e controle

\begin{tabular}{|c|c|c|c|c|c|c|c|}
\hline & \multicolumn{3}{|c|}{ Paralisia Cerebral } & \multicolumn{3}{|c|}{ Controle } & \multirow[t]{2}{*}{$\mathrm{p}$} \\
\hline & Mínima & Média/DP & Máxima & Mínima & Média/DP & Máxima & \\
\hline DiP pré & 288 & $681 \pm 256,5$ & 927 & 795 & $1253,5 \pm 320,5$ & 1662 & $0,003 *$ \\
\hline DiP pós & 465 & $930,7 \pm 264,5$ & 1242 & 795 & $1253,5 \pm 320,5$ & 1662 & $0,046^{*}$ \\
\hline $\mathrm{VO}_{2}$ pré & $-4,4$ & $3,9 \pm 5,8$ & 9,3 & 6,5 & $16,7 \pm 7,1$ & 25,8 & $0,003^{*}$ \\
\hline $\mathrm{VO}_{2}$ pós & 0,9 & $9,7 \pm 5,5$ & 16,5 & 6,5 & $16,7 \pm 7,1$ & 25,8 & $0,046^{*}$ \\
\hline PASi pré & 90 & $100 \pm 10,7$ & 120 & 90 & $101,2 \pm 9,9$ & 120 & $0,738^{\mathrm{NS}}$ \\
\hline PASi pós & 80 & $88,7 \pm 8,3$ & 100 & 90 & $101,2 \pm 9,9$ & 120 & $0,021 *$ \\
\hline PADi pré & 60 & $66,2 \pm 10,6$ & 90 & 60 & $68,7 \pm 6,4$ & 80 & $0,251^{\mathrm{NS}}$ \\
\hline PADi pós & 50 & $60 \pm 9,2$ & 70 & 60 & $68,7 \pm 6,4$ & 80 & $0,061^{\mathrm{NS}}$ \\
\hline PASf pré & 90 & $110 \pm 11,9$ & 130 & 110 & $121,2 \pm 9,9$ & 140 & $0,055^{\mathrm{NS}}$ \\
\hline PASf pós & 80 & $102,5 \pm 15,8$ & 120 & 110 & $121,2 \pm 9,9$ & 140 & $0,028^{*}$ \\
\hline PADf pré & 60 & $68,7 \pm 8,3$ & 80 & 60 & $71,2 \pm 8,3$ & 80 & $0,54^{\mathrm{Ns}}$ \\
\hline PADf pós & 50 & $63,7 \pm 11,9$ & 80 & 60 & $71,2 \pm 8,3$ & 80 & $0,172^{\mathrm{NS}}$ \\
\hline
\end{tabular}

Mann Whitney não paramétrico *: diferenças estatisticamente significativas $\mathrm{p}<0,05$, NS: não significativo. $\mathrm{DiP}=$ distância percorrida; $\mathrm{VO}_{2}=$ consumo máximo de oxigênio; $\mathrm{PASi}=$ pressão arterial sistólica inicial; $\mathrm{PADi}=$ pressão arterial diastólica inicial; $\mathrm{PASf}=$ pressão arterial sistólica final; PADf= pressão arterial diastólica final. 
Por meio da comparação entre os valores do pós-treinamento e os resultados obtidos na avaliação após o período de destreinamento, pode-se constatar redução significativa apenas do $\mathrm{VO}_{2}$ máx (Tabela 4).

Tabela 4 - Mínima, média, desvio padrão e máxima dos valores de DiP, VO2, PASi, PADi, PASf e PADf no póstreinamento e reavaliação após período de destreinamento (12 semanas) do grupo com paralisia cerebral

\begin{tabular}{|c|c|c|c|c|c|c|c|}
\hline & \multicolumn{3}{|c|}{ Pós-tratamento } & \multicolumn{3}{|c|}{ Reavaliação } & \multirow[t]{2}{*}{$\mathrm{p}$} \\
\hline & Mínima & Média/DP & Máxima & Mínima & Média/DP & Máxima & \\
\hline DiP & 465 & $905,6 \pm 275,1$ & 1242 & 498 & $817,7 \pm 198,6$ & 1080 & $0,063^{\mathrm{NS}}$ \\
\hline $\mathrm{VO}_{2}$ & 0,9 & $9,2 \pm 5,7$ & 16,5 & 0,1 & $7,0 \pm 4,4$ & 12,9 & $0,018^{*}$ \\
\hline PASi & 80 & $87,1 \pm 7,6$ & 100 & 80 & $90 \pm 8,2$ & 100 & $0,157^{\mathrm{NS}}$ \\
\hline PADi & 50 & $58,6 \pm 9,0$ & 70 & 50 & $61,4 \pm 6,9$ & 70 & $0,317^{\mathrm{NS}}$ \\
\hline PASf & 80 & $100 \pm 15,3$ & 120 & 100 & $105,7 \pm 5,3$ & 110 & $0,206^{\mathrm{NS}}$ \\
\hline PADf & 50 & $64,3 \pm 12,7$ & 80 & 50 & $61,4 \pm 6,9$ & 70 & $0,705^{\mathrm{NS}}$ \\
\hline
\end{tabular}

Wilcoxon não paramétrico *: diferenças estatisticamente significativas $\mathrm{p}<0,05$, NS: não significativo. DiP $=$ distância percorrida; $\mathrm{VO}_{2}=$ consumo máximo de oxigênio; $\mathrm{PASi}=$ pressão arterial sistólica inicial; $\mathrm{PADi}=$ pressão arterial diastólica inicial; $\mathrm{PASf}=$ pressão arterial sistólica final; PADf= pressão arterial diastólica final.

\section{Discussão}

Existem poucos estudos que examinem a efetividade desse tipo de treinamento nesta população, pois antigamente se acreditava que esse tipo de intervenção aumentaria o tônus, padrões anormais de movimento e o gasto energético. Hoje em dia já se sabe que essa população pode se beneficiar com o treinamento aeróbico (11,14-16). Van Den Berg-Emons et al. (17) implementaram um programa de treinamento de exercício aeróbico, quatro vezes por semana durante nove meses, em um grupo de crianças com paralisia cerebral, que resultou na melhora de 35\% da capacidade aeróbica quando comparada com o grupo controle.

Os resultados mostram redução da pressão arterial sistêmica (PAS) do grupo com paralisia cerebral no pós-treinamento, evidenciando efeitos positivos sobre o condicionamento cardiorrespiratório. Potempa (18) sugere que exercícios submáximos diminuem a frequência cardíaca e a pressão arterial. Por meio da comparação entre grupos constata-se, através da PAS, que no pré-treinamento ambos apresentavam nível de condicionamento semelhante, com surgimento de diferença no pós treinamento. Essa diferença justifica-se pela melhora do condicionamento cardiorrespiratório no grupo com PC e pela não participação do treinamento do grupo controle. Sugere também, entre outros fatores, que os sujeitos sem alterações neurológicas podem fazer parte do grupo de população sedentária.

Em estudos com adolescentes brasileiros encontra-se porcentagem de sedentarismo que varia entre 42\% e 94\% (19-21). Foi realizado um estudo com 960 adolescentes residentes na cidade de Pelotas, que responderam um questionário relacionado à sua atividade física, e o resultado mostrou que 39\% foram considerados sedentários (22). Evidências sugerem aumento na prevalência de hipertensão arterial (HA) em crianças nas últimas décadas, e estudos epidemiológicos realizados no Brasil mostram uma porcentagem de $0,8 \%$ à $8,2 \%$ de crianças com HA $(23,24)$.

$\mathrm{Na}$ distância percorrida houve melhora significativa após treinamento no grupo com PC, porém os valores não se igualaram aos obtidos pelo grupo controle. Os indivíduos com paralisia cerebral apresentam 
diminuição do controle muscular seletivo, alteração nas fibras musculares, padrões de ativação muscular alterados, fraqueza muscular, tônus anormal e baixa tolerância ao exercício físico (25, 26), o que leva essa população apresentar velocidade da marcha mais lenta, maior gasto energético e déficit do condicionamento cardiorrespiratório $(27,28)$. Nesses indivíduos o gasto energético necessário para realizar a deambulação de rotina é elevado em torno de 1,5 a 2 vezes (5).

A mesma situação ocorre com o $\mathrm{VO}_{2}$ máx, por ele ser uma medida indireta calculada a partir da distância percorrida. Um estudo realizado por Taka-aki Shinohara et al. (14), com crianças com paralisia cerebral espástica, durante 20 semanas, com cicloergômetro, observou aumento significativo na captação de oxigênio $\left(\mathrm{VO}_{2}\right)$. Existem evidências na literatura demonstrando que após o treinamento aeróbico os pacientes apresentam melhora na capacidade ventilatória e aumento da capacidade oxidativa do músculo diafragma, o que poderia ser responsável pelo aumento do $\mathrm{VO}_{2}(15)$.

O destreinamento em crianças e adolescentes com paralisia cerebral é pouco investigado. Blundell et al. (29) realizaram uma reavaliação após oito semanas do término de treinamento aeróbico e observaram que as melhoras foram mantidas; o mesmo ocorreu com o estudo de Darrah et al. (30), após dez semanas do término do treinamento. Neste estudo, o período de destreinamento foi maior que os citados anteriormente (12 semanas), e houve perda significativa apenas no consumo máximo de oxigênio.

\section{Considerações finais}

Com base nos achados do presente estudo, pode-se concluir que o período de 24 sessões de treinamento foi suficiente para obter modificações significativas nas variáveis analisadas, mas não para alcançar índices semelhantes aos atingidos por crianças sem alterações neurológicas. As 12 semanas sem treinamento ocasionaram regressão dos parâmetros cardiorrespiratórios, sugerindo que este período não deve ser prolongado. Sendo assim, a população com paralisia cerebral hemiparética e diparética espástica beneficia-se do treinamento aeróbico, devendo este ser incluído nos programas de reabilitação.

A partir deste estudo, pesquisas podem ser realizadas para responder perguntas ainda não esclarecidas: quais fatores mais se relacionam com a velocidade da melhora do condicionamento cardiorrespiratório em crianças com paralisia cerebral (tono muscular, força muscular, comprimento muscular)? Como se comportam as crianças sem déficits neurológicos quando submetidas a treinamento similar e quais as diferenças com a evolução das crianças com PC? Qual o período máximo de destreinamento que não ocasiona perda do condicionamento cardiorrespiratório em crianças com PC?

\section{Referências}

1. Sankar C, Mundkur N. Cerebral Palsy - definition, classification, etiology and early diagnosis. Indian J Pediatr. 2005;72(10):865-8.

2. Moura EA, Campos SPA. Fisioterapia: aspectos clínicos e práticos da reabilitação. In: Gianni MAC. Paralisia cerebral: aspectos clínicos. São Paulo: Artes Médicas; 2005. p. 13-6.

3. Maltais DB, Pirrynowst MR, Galea VA, Bar-Or O. Physical activitiy level is associated with the $\mathrm{O}_{2}$ cost of walking in cerebral palsy. Med Sci Sports Exerc. 2005;37(3):347-53.

4. Mota AP, Pereira JS. Influência da fisioterapia nas alterações motoras em crianças com paralisia cerebral. Fisioter Bras. 2006;7(3):25-31.

5. Teixeira-Salmela LF, Lima RCM, Morais LAO, Goulart F. Assimetria e desempenho funcional em hemiplégicos crônicos antes e após programa de treinamento em academia. Rev Bras Fisioter. 2005;9(2):227-33.

6. Teixeira-Salmela LF, Oliveira ESG, Santana EGS, Resende GP. Fortalecimento muscular e condicionamento físico em hemiplégicos. Acta Fisiátrica. 2000;7(3):108-18. 
7. Macko RF, DeSouza CA, Tretter LD, Silver KH, Smith GV, Anderson PA, et al. Treadmill aerobic training reduces the energy expenditure and cardiovascular demands of hemiparetic gait in chronic stroke patients. Stroke. 1997;28(2):326-30.

8. Teixeira-Salmela LF, Silva PC, Lima RCM, Augusto ACC, Souza AC, Goulart F. Musculação e condicionamento aeróbio na performance funcional de hemiplégicos crônicos. Acta Fisiátrica. 2003;10(3):54-60.

9. Brown DW, Heath GW, Balluz L, Giles WH, Ford ES, Mokdad AH, et al. Associations between physical activity dose and health-related quality of life. Med Sci Sports Exerc. 2004;36(5):890-6.

10. Rimmer JH, Wang E. Aerobic exercise training in stroke survivors. Top Stroke Rehabil. 2005;12(1):17-30.

11. Fowler EG, Knutson LM, DeMuth SK, Sugi M, Siebert K, Simms V, et al. Pediatric endurance and limb strengthening for children with cerebral palsy (PEDALS) - a randomized controlled trial protocol for a stationary cycling intervention. BMC Pediatr. 2007;7(14):1-9.

12. Kiss MAPBM. Avaliação em educação física: aspectos biológicos e educacionais. São Paulo: Manole; 1987.

13. Kisner C, Colby LA. Exercícios terapêuticos: fundamentos e técnicas. São Paulo: Manole. 1998.

14. Shinohara TA, Suzuki N, Oba M, Kawasumi M, Kimizuka M, Mita K. Effect of exercise at the point for children with cerebral palsy. Bull Hosp Jt Dis. 2002-2003;61(1-2):63-7.

15. Unnithan VB, Katsimanis G, Evangelinou C, Kosmas C, Kandrali I, Kellis E. Effect of strength and aerobic training in children with cerebral palsy. Med Sci Sports Exerc. 2007;39(11):1902-9.

16. Schlough K, Nawoczenski D, Case LE, Nolan K, Wigglesworth JK. The effects of aerobic exercise on endurance, strength, function and self-perception in adolescents with spastic cerebral palsy: a report of three case studies. Pediatr Phys Ther. 2005;17(4):234-50.

17. Van Den Berg-Emons RJG, Van Baak MA, Speth L, Saris WH. Physical training in school children with spastic cerebral palsy: effects on daily activity, fat mass and fitness. Int J Rehabil Res. 1998;21(2):179-94.

18. Potempa K, Braum LT, Tinknell T, Popovich J. Benefits of aerobic exercise after stroke. Sports Med. 1996; 21(5):337-46.

19. Guedes, DP, Guedes, JERP, Barbosa, DS, Oliveira, JA. Níveis de prática de atividade física habitual em adolescentes. Rev Bras Med Esporte. 2001;7(6):187-99.

20. Pinho RA, Petroski EL. Nível habitual de atividade física e equilíbrio energético de adolescentes. Rev Bras Ativ Fís Saúde. 1999;4:5-16.

21. Silva RCR. Nível de atividade física em adolescentes do Município de Niterói, Rio de Janeiro, Brasil. Cad Saúde Pública. 2000;16:1091-7.

22. Oehischiaeger MHK, Pinheiro RT, Horta B, Gelatti C, San’Tana P. Prevalência e fatores associados ao sedentarismo em adolescentes de área urbana. Rev Saúde Pública. 2004;38(2):157-63.

23. Nogueira PCK, Costa RF, Cunha JSN, Fisserg M. Pressão arterial elevada em escolares de Santos - relação com a obesidade. Rev Assoc Méd Bras. 2007;53(5):426-32.

24. Silva MAM, Rivera IR, Souza MGB, Carvalho ACC. Medida da pressão arterial em crianças e adolescentes: recomendações das diretrizes de hipertensão arterial e prática médica atual. Arq Bras Cardiol. 2007;88(4): 491-5.

25. Norma JF, Bossman S, Gardner P, Moen C. Comparison of the energy expenditure index and oxygen consumption index during self-paced walking in chidren with spastic diplegia cerebral palsy and children whithout physical disabilities. Pediatr Phys Ther. 2004;16(4):206-11. 
26. Patikas D, Wolf SI, Mund K, Armbrust P, Schuster W, Doderlein L. Effects of a postoperative strength-training program on the walking ability of children with cerebral palsy: a randomized controlled trial. Arch Phys Med Rehabil. 2006;87(5):619-26.

27. Maltais D, Bar-Or O, Pirrynowski M, Galea V. Reapeated treadmill walks affect physiologic responses in children with cerebral palsy. Med Sci Sports Exerc. 2003;35(10):1653-61.

28. Maltais D, Wilk Boguslaw W, Unnithan V, Bar-Or O. Responses of children palsy to treadmill walking exercise in the heat. Med Sci Sports Exerc. 2004;36(10):1674-81.

29. Blundell SW, Sheperd RB, Dean CM, Adams RD. Fucntional strength training in cerebral palsy: a pilot study of a group circuit training class for children aged 4-8 years. Clin Rehabil. 2003;17(1):48-57.

30. Darrah J, Wessel J, Nearinburg P, O' Connor M. Evaluation of a community fitness program for adolescents with cerebral palsy. Pediatr Phys Ther. 1999;11(1):18-23.

Recebido: 06/11/2009

Received: 11/06/2009

Aprovado: $23 / 03 / 2010$

Approved: 03/23/2010

Revisado: 06/05/2010

Reviewed: 05/06/2010 